\title{
Bandwidth \& Modulation Techniques Under Study by NASA
}

\author{
J. C. Morakis \& W. H. Miller \\ NASA \\ Goddard Space Flight Center \\ Greenbelt, MD 20771
}

\begin{abstract}
As NASA programs demand higher data rates, the need for more bandwidthefficient high-rate modulation schemes grows. This is of special interest to NASA if they are to reduce future users' required communication bandwidth and minimize spacecraft contact time to the ground stations. Currently, the Tracking \& Data Relay Satellite System (TDRSS) KSA channel operates with Quadrature Phase Shift Keying and a rate 1/2 convolutional code. One of the strongest candidates for increasing data throughput without increasing the required bandwidth is Octal Phase Shift Keying (8PSK). Eight phase Trellis Coded Modulation using a rate $2 / 3$ convolutional code, for example, will double the throughput without any change in the bandwidth requirements when compared to the current system. Other coding schemes can be employed for higher throughput or coding gain depending on the program requirements.
\end{abstract}

In order to ensure that NASA can support high data rate programs through TDRSS, the errorrate performance of 8PSK operated through the telecommunication link at 450 Mega-bits per second (Mbps) was measured by the New Mexico State University (NMSU)* [1][2]. Octal Phase-Shift keying was chosen because it has proven to be the strongest candidate for upcoming systems, exhibiting a higher

\footnotetext{
* NASA Grant \#NAG51491
}

information rate than QPSK for the same transmition bandwidth.

Channel measurements were made at the White Sands Ground Terminal (WSGT) and are summarized.

\section{TABLE OF CONTENTS}
1. INTRODUCTION
2. QPSK
3. UNCOIED 8PSK
4. TRELLIS CODED MODULATION
5. CONCLUSIONS

\section{INTRODUCTION}

The growth of technology, especially in the area of multispectral band imaging instruments, has resulted in a demand for very high data rates. For example, the Landsat 8 Imager data will be in the order of 1 Gbps. Presently the Tracking and Data Relay Satellite System (TDRSS) Ku band Single Access (KSA) channel can operate at 300 Mbps data rates using Quadrature Phase Shift Keying (QPSK) and a rate $1 / 2$ convolutional code; this is equivalent to an information rate of $150 \mathrm{Mbps}$. 
NASA's future data-rate requirements for Earth Imaging Instruments are outlined in Table 1. It can be seen that the future requirements are beyond the present capabilities. Studies were conducted to ensure that NASA will be able to support these high data rate programs while using the existing TDRS system. Study results indicate that lossless data compression combined with bandwidth-efficient modulation techniques will enable data rates of 1 Gbps to be transferred via the TDRSS.

\section{QPSK}

The TDRSS is presently using both PSK and QPSK. There are two channels: the in-phase (I) and the quadrature phase (Q) (see Figure 1).
Each channel is PSK and one channel is 90 degrees out of phase from the other, thus resulting in a channel equivalent to QPSK.

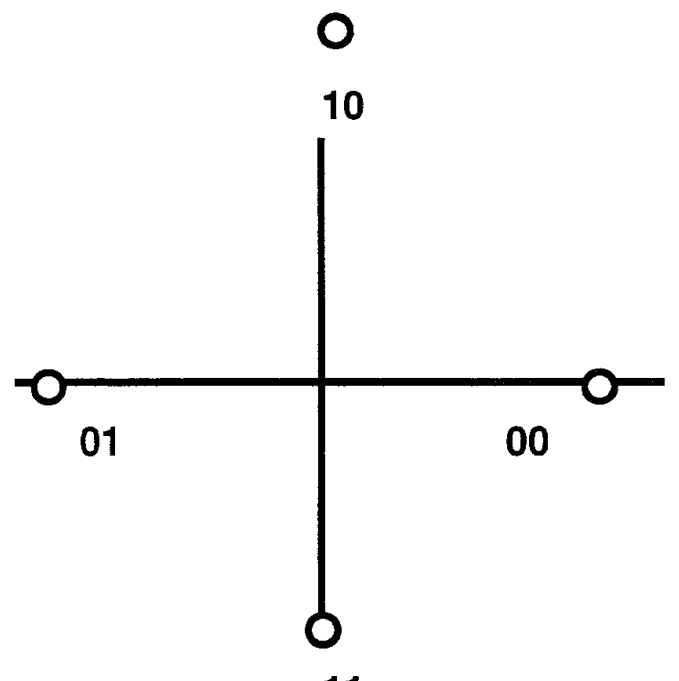

11

Figure 1. QPSK Signal

Table 1. NASA's Data Rate Requirements

Real Time Processing of Imaging Arrays Relationship between Spatial Resolution and Data Rate

For One Spectral Band Assuming 185k FOV and An Earth Viewing Altitude of 700 km, 8 Bits/Pixel

\begin{tabular}{|c|c|c|c|c|c|c|}
\hline & $\begin{array}{l}\text { IFOV } \\
(\mathrm{m})\end{array}$ & $\begin{array}{l}\text { \#Det. } \\
\text { per } \\
\text { FOV }\end{array}$ & $\begin{array}{l}\text { Integ. } \\
\text { Time } \\
\text { (Dwell) } \\
\text { (msec.) }\end{array}$ & $\begin{array}{l}\text { Pixel } \\
\text { Rate } \\
\text { (pps) }\end{array}$ & $\begin{array}{l}\text { Pixel } \\
\text { Period } \\
\text { ( } \mu \text { sec.) }\end{array}$ & $\begin{array}{c}\text { Serial Data } \\
\text { Rate per } \\
\text { Spectral Band } \\
\text { (b/sec.) }\end{array}$ \\
\hline & 120 & 1542 & 17.7 & $87 \mathrm{~K}$ & 11.5 & $695.7 \mathrm{~K}$ \\
\hline \multirow{3}{*}{ Multi Spectral Scanner } & 80 & 2313 & 11.8 & $196 \mathrm{~K}$ & 5.1 & $1.57 \mathrm{M}$ \\
\hline & 60 & 3083 & 8.9 & $347.7 \mathrm{~K}$ & 2.9 & $2.78 \mathrm{M}$ \\
\hline & 40 & 4625 & 5.9 & $782.4 \mathrm{~K}$ & 1.3 & $6.26 \mathrm{M}$ \\
\hline \multirow[t]{4}{*}{ Thematic Mapper } & 30 & 6167 & 4.4 & $1.39 \mathrm{M}$ & 0.72 & $11.13 \mathrm{M}$ \\
\hline & 20 & 9250 & 2.9 & $3.13 \mathrm{M}$ & 0.32 & $25.0 \mathrm{M}$ \\
\hline & 15 & 12334 & 2.2 & $5.56 \mathrm{M}$ & 0.179 & $44.5 \mathrm{M}$ \\
\hline & 10 & 18500 & 1.47 & $12.5 \mathrm{M}$ & 0.08 & $100.00 \mathrm{M}$ \\
\hline Panchromatic Imager & 5 & 37000 & 0.73 & $50.0 \mathrm{M}$ & 0.02 & 400.0M \\
\hline
\end{tabular}


The performance of this channel, theoretical and measured, for the TDRSS Ku-band is given in Figure 2.

\section{UNCODED 8PSK}

Eight PSK can be thought of as the combination of two QPSK constellation sets.

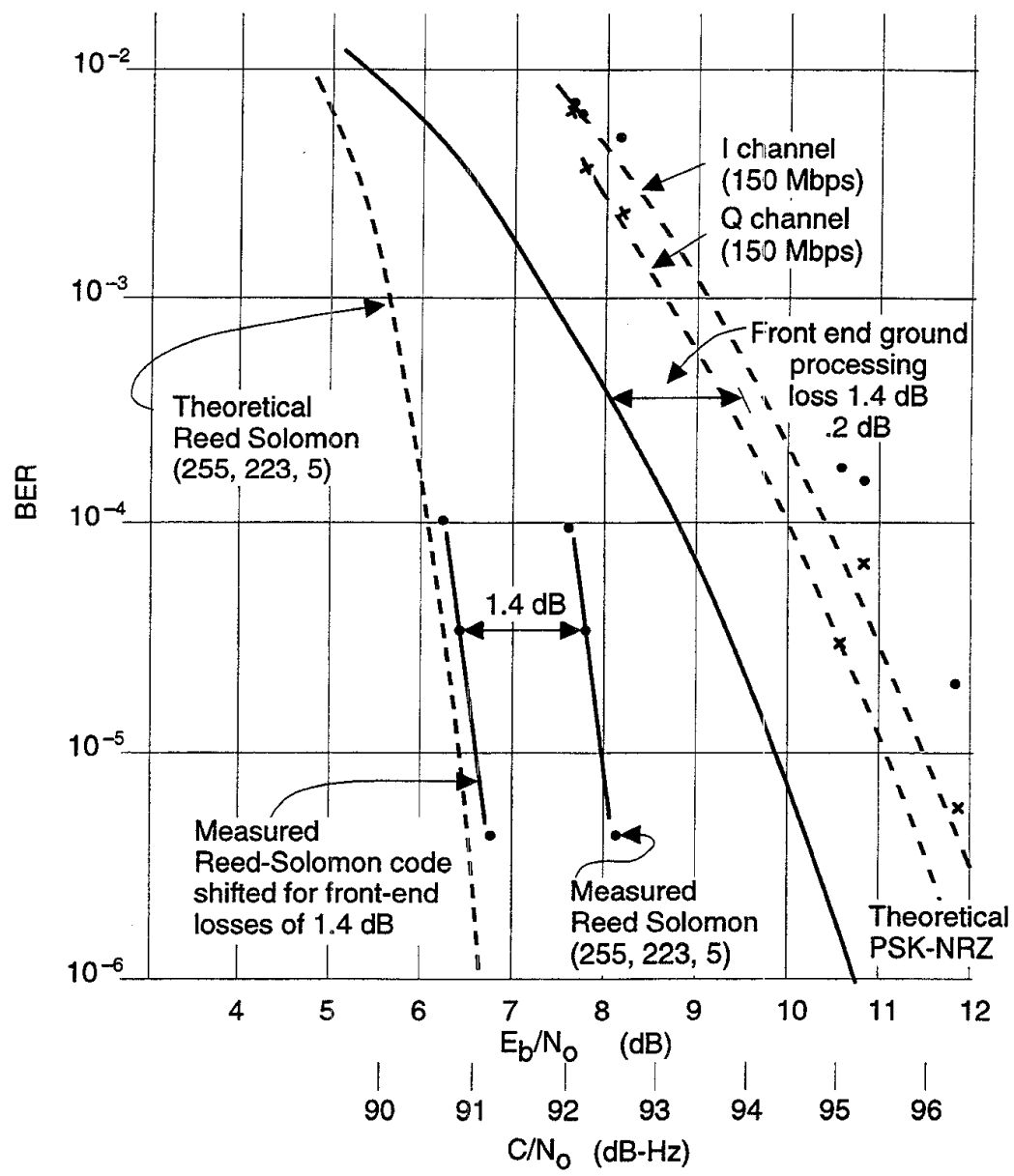

Figure 2. TDRSS QPSK Performance

The tests in Figure 2 utilized the TDRSS at 300 Mbps (150 Mbps on I and Q), and were run with and without Reed-Solomon (RS[255,223]) codex. The theoretical carrier-to-noise ratio $\left(\mathrm{C} / \mathrm{N}_{\mathrm{o}}\right)$ is $94.4 \mathrm{~dB}-\mathrm{Hz}$ or $\mathrm{E}_{\mathrm{b}} / \mathrm{N}_{\mathrm{o}}=9.6 \mathrm{~dB}$ for $\mathrm{a}$ Bit Error Rate (BER) of $10^{-5}$. Note that the Reed Solomon code provides a coding gain of $3.4 \mathrm{~dB}$ at $10^{-5}$ BER with $12.5 \%$ overhead.
Figure 3 (a) shows an 8PSK signal set. Since each symbol of the 8PSK is represented by 3 bits, and the required bandwidth is related to the symbol rate rather than the bit rate, it follows that the binary data transmitted, using 8PSK, through the same bandwidth is triple that of PSK and 1.5 times that of QPSK. 




(a) 8-PSK Signal Set

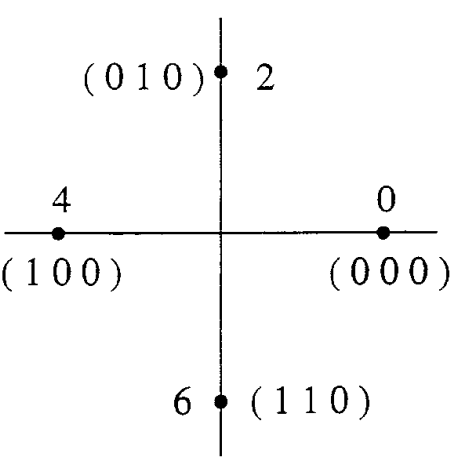

(b) QPSK

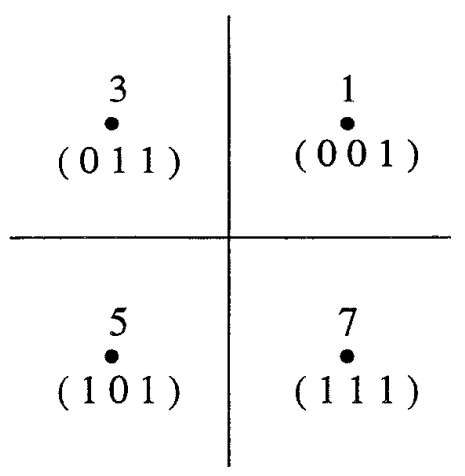

(c) QPSK

Figure 3. An 8PSK Signal Set

Currently the TDRSS employs both PSK and QPSK in the KSA channel at maximum rates of $300 \mathrm{Mbps}$ (150 Mega Symbols Per Second [Msps]). In theory this channel should support 8PSK at 150 Msps rates, which is equivalent to $450 \mathrm{Mbps}$.

In February 1995 NMSU and LORAL WDL conducted a series of high-speed tests with various modulation schemes over the TDRSS KSA channel and demonstrated the ability to transmit data through the TDRSS, utilizing 8PSK, at $450 \mathrm{Mbps}$ with bit error rates of $10^{-3}$ at $\mathrm{Eb}_{\mathrm{b}} / \mathrm{N}_{\mathrm{o}}=12.5 \mathrm{~dB}$ (Figure 4 curve $\mathrm{A}$ ) using pre-equalization to compensate for phase nonlinearity. The pre-equalization approach was developed at NMSU using the Volterra technique [3].

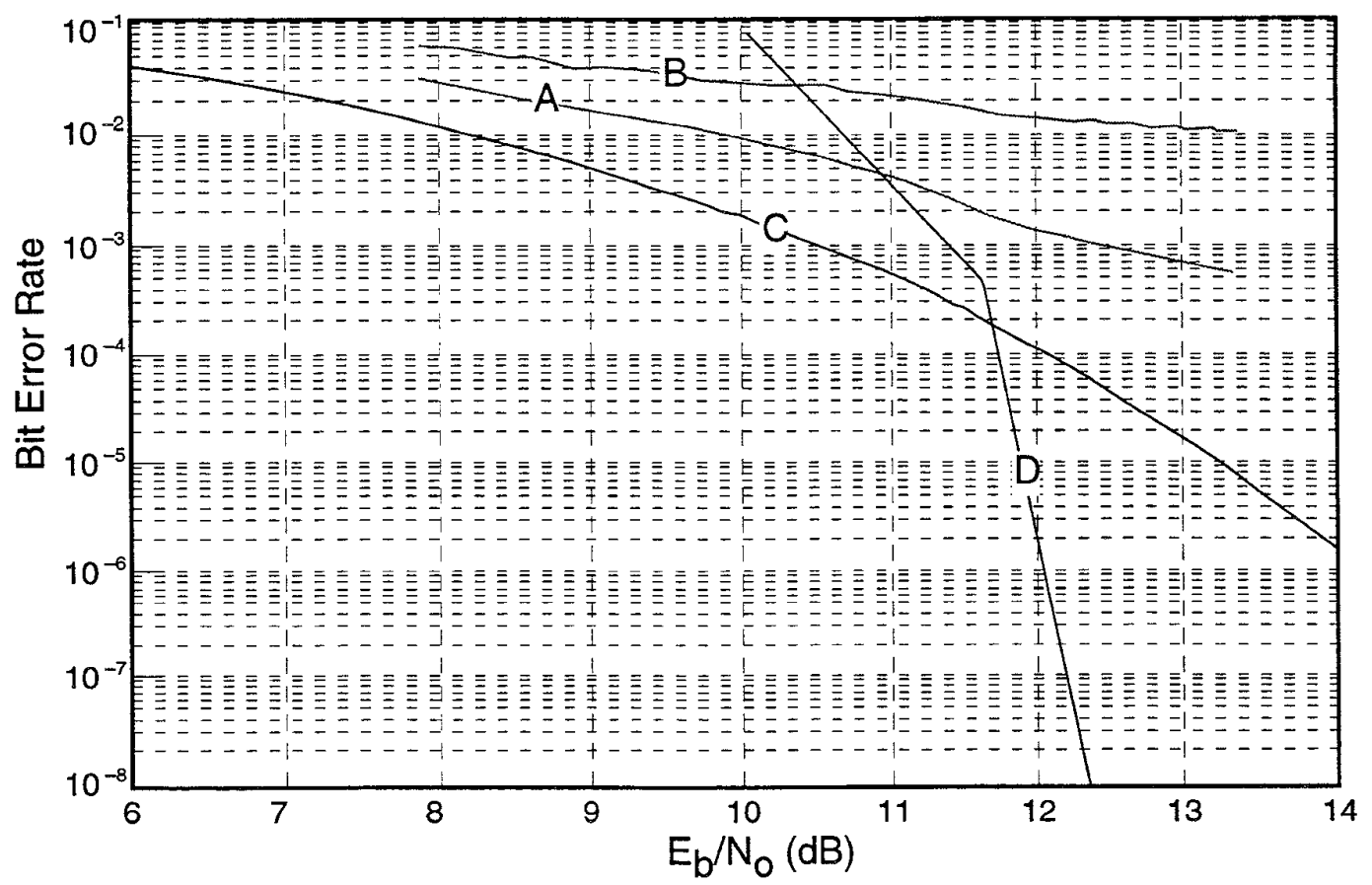

Figure 4. 450 Mbps 8PSK Test Results 
Performance without pre-equalization was unacceptable (Figure 4 curve B). Also shown in Figure 4 is the expected performance when the Reed-Solomon $(255,223)$ block code is applied. The resultant data rate is $393 \mathrm{Mbps}$ with BER of $10^{-7}$ at $E_{b} / N_{0}=12 \mathrm{~dB}$.

Figure 5 shows the phase plane distribution of 8PSK constellation with and without preequalization.
The studies pursued at GSFC and the Universities of Notre Dame and Hawaii include both TCM and BCM; however, because of the insensitivity of the TDRSS to amplitude variations we confined studies to MPSK for now.

In the previous section it was shown that the use of 8PSK triples the information rate of PSK. By examining Figure 3 curve $A$ it can be

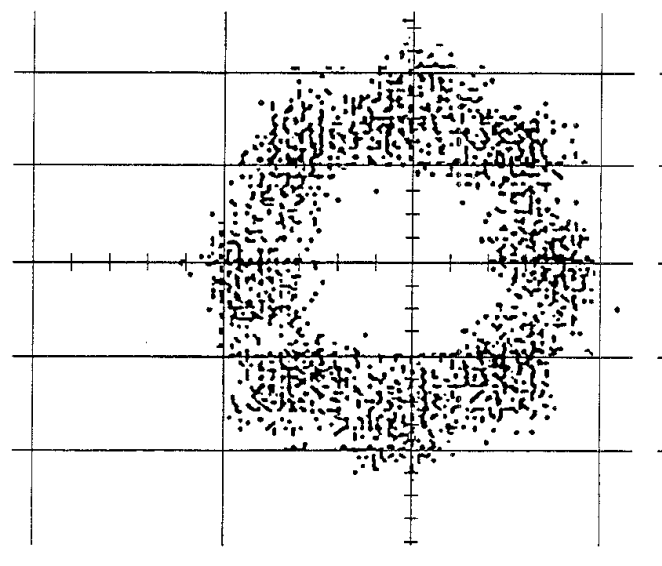

No pre-equalization

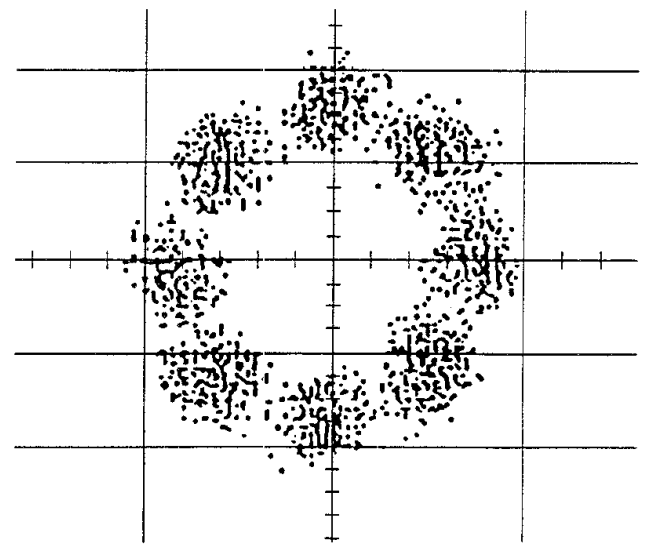

Pre-equalization

Figure 5. Phase Plane Distribution

\section{TRELlis CODED MOdULATION}

The basic concept of the Trellis Coded Modulation technique is to perform coding onto an expanded modulation signal set. Coding is used to form structured signal sequences with large minimum Euclidean distance.

Coded modulations can be categorized as Trellis Coded Modulation (TCM) or Block Coded Modulation (BCM) depending on the coded structure. They can also be constant envelope, Multiple Phase Shift Keying (MPSK), or lattice-type (QASK). seen that, as the number of bits per symbol $\left(\log _{2} M\right)$ in an MPSK increases, the angle of the decision regions decreases, thus decreasing the tolerance to noise, which becomes a fuzzball centered at each symbol point (Figure 5). If we operate in a constant signalto-noise environment we need to combat this increase in error rate by using TCM. TCM is the combination of MPSK and coding using trellis decoding techniques. Figure 6 shows the Convolutional $(3,2,6)$ TCM encoder [4], and Figure 7 shows the $(6,5,4) 2 \times 8$ PSK encoder [5] with a spectral efficiency of 2.5 bits/symbol used by the NMSU in the tests through the TDRSS at the White Sands 


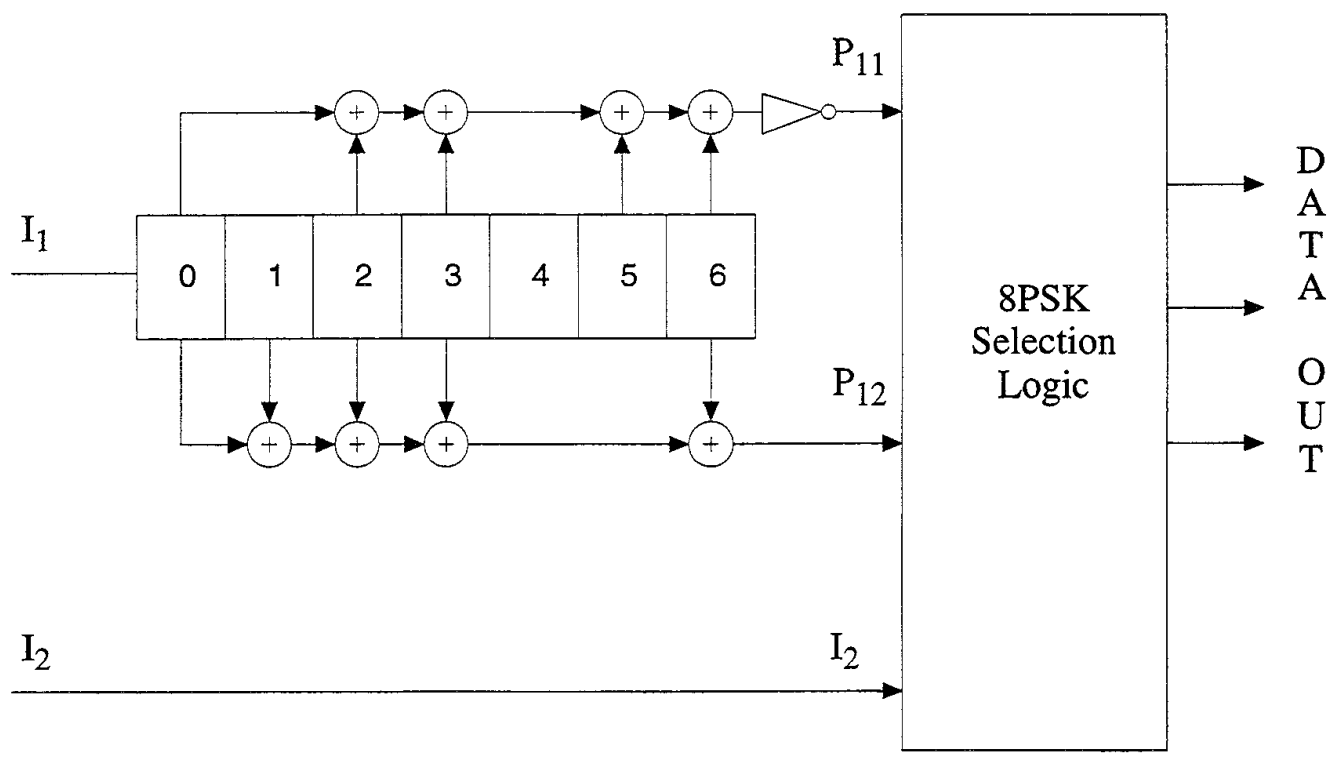

Figure 6. TCM Using $(3,2,6)$ Convolutional Encoder \& 8PSK

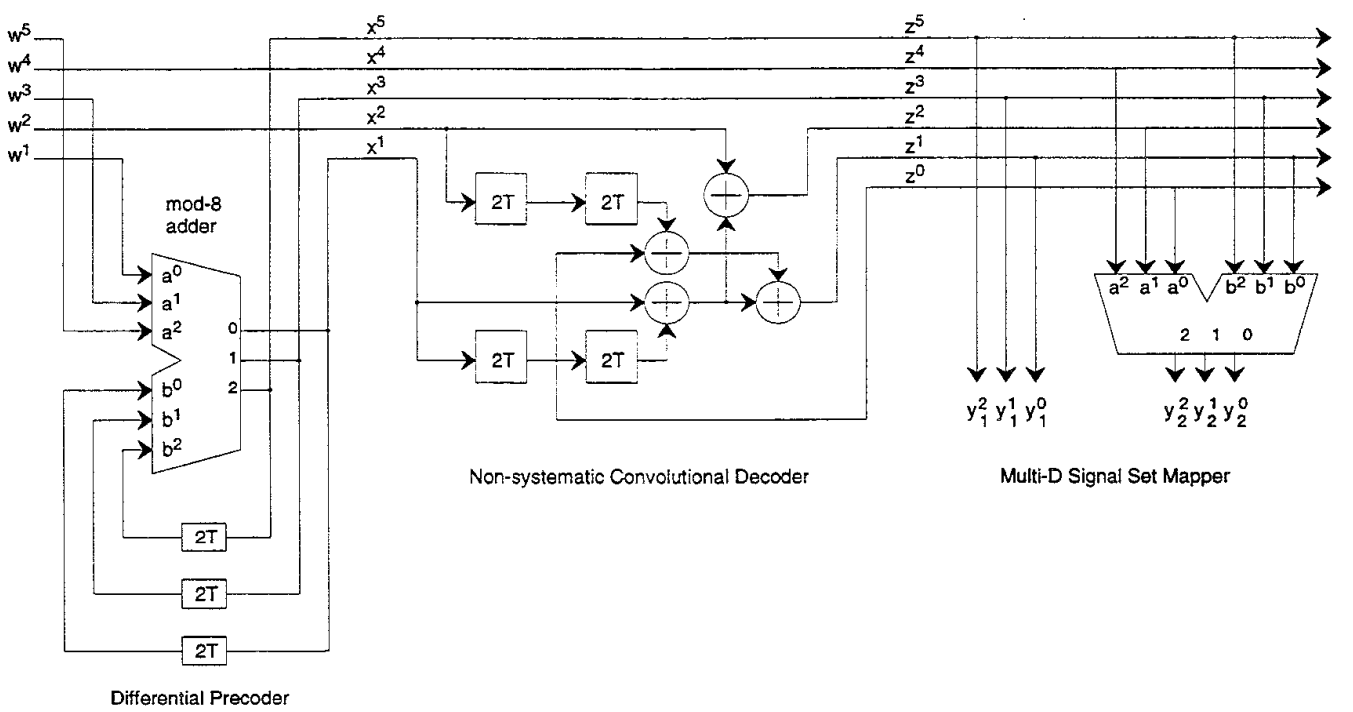

Figure 7. Block Coded Modulation Using a $(6,5,4)$ Code 


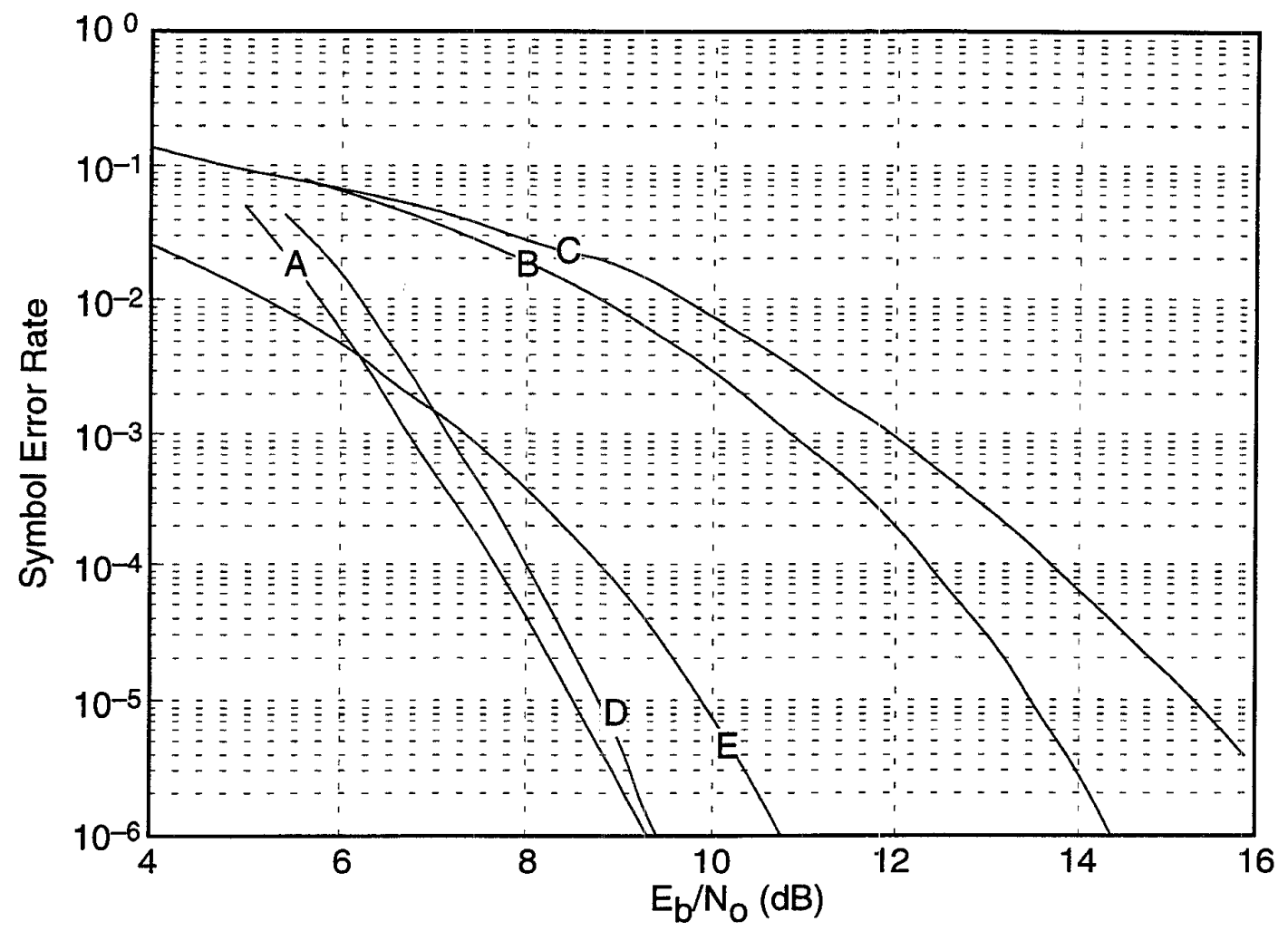

Figure 8. 8PSK TCM Test Results

Ground Terminal (WSGT). The 1992 tests using both of these codes at the S-band Single access (SSA) and KSA channels were conducted at 2 MBPS. The results are shown in Figure 8. The University of Notre Dame rate $5 / 6$ convolutional code had a phase invariant property. The correct phase sequence is required by the decoding process. The $(6,5,4)$ code inherently selects the correct phase sequence by using differential modulation techniques; this is accomplished at the cost of $.07 \mathrm{~dB}$. The tradeoff is the ability to transmit at $2.5 \mathrm{bits} / \mathrm{symbol}$ versus 2.0 bits/symbol for uncoded QPSK, and a BER of $10^{-5}$ with $1.5 \mathrm{~dB}$ less signal/noise can be achieved.

\section{CONCLUSIONS}

We have demonstrated that for a given BER of $10^{-5}$.when combining 8-phase PSK Modulation and a rate $(6,5,4)$ coding technique, a coding gain of $1 \mathrm{~dB}$ is obtainable with a bandwidth efficiency of 2.5 bits/hertz as compared to 2 bits/hertz for QPSK and $1 \mathrm{bit} /$ hertz for PSK.

When combining these higher order modulation techniques such as 8-phase PSK with Lossless Data Compression techniques (reference [6] under development by NASA) bandwidth efficiency of close to 6 bits/hertz will be achievable in the near future. 


\section{REFERENCES}

[a] William P. Osborne and Jery Stolaczyk, Modification of the TDRSS High Rate Demodulator for 8-PSK Operation, Technical Report NMSU-ECE-92-010, New Mexico: New Mexico State University, May 1992.

[2] Micheal D. Ross, High Speed Architecture for the Decoding of Trellis-Coded Modulation, Doctoral Dissertation, New Mexico: New Mexico State University, December 1992.

[3] A. Gutierrez and W. E. Wyan, "Performance of Adaptive Volterra Equalizers on Non-Linear Band Limited Satellite Channels," International Conf. Comm. January $1985,408-492$.

[4] Andrew J. Viterbi, Jack K. Wolf, Ephraim Zehavi, and Roberto Padovani, "A Pragmatic Approach to Trellis-Coded Modulation," IEEE Communications Magazine, Vol 27, No. 7, July 1989, 11-19.
[5] Steven S. Pietrobon, Trellis Coding with Multidimensional Signal Sets and Rotationally Invariant Trellis Codes, Doctoral Disseretation, Indiana: University of Notre Dame, December 1990.

[6] Pen-Shu Yeh, Warner H. Miller, and Steve Hou, "Overview of NASA's Lossless Compression Technology: Development and Applications," Procedings of MILCOM'95, San Diego, November 6-8, 1995.

James C. Morakis received a B.S. in Electrical Engineering from the University of the City of New York, an M.S. from Columbia University, and Ph.D. from the University of Maryland. $\mathrm{He}$ worked in communications at Bell Telephone Laboratories, Bendix Systems, and Martin Marietta Corporation. For the past 30 years he has worked at NASA/Goddard Space Flight Center in the areas of satellite, telemetry, telecommand, coding, and trelliscoded modulation techniques. 